\title{
Glycerosomes: Advanced Liposomal Drug Delivery System
}

\author{
PRIYA GUPTA*, RUPA MAZUMDER AND SWARUPANJALI PADHI \\ Department of Pharmaceutics, Noida Institute of Engineering and Technology (Pharmacy Institute), Plot no. 19, Knowledge \\ Park-II, Greater Noida-201 306, Uttar Pradesh, India
}

\section{Gupta et al.: Glycerosomal Drug Delivery Systems}

\begin{abstract}
Although liposomes have proved to be versatile in the field of drug delivery, there are still some issues that need to be addressed mainly problems related to entrapment, penetration, fluidity and stability. Glycerosomes represent a novel drug delivery systems composed of phospholipids, water and glycerol in varying amounts. Glycerosomes are upgraded versions of liposomes meant for topical and transdermal drug delivery. In these preparations, glycerol acts as an edge activator and penetration enhancer. These drug delivery systems manifest improved stability, fluidity, entrapment and penetration in comparison to conventional liposomes. Increasing the concentration of glycerol by 10,20 or $30 \%$ leads to a drastic increase in glycerosome stability. This article is a detailed review of glycerosomes focusing on methods of preparation, evaluation, applications, advantages and disadvantages based on studies reported so far. In this review article, how the structure of liposomes can be modified by the addition of glycerol and how this modified structure can be used to address the problems related to liposomes is discussed. The influence of increasing glycerol concentration on the nature of glycerosomes that lead to an increase in both entrapment and encapsulation of a drug while causing slower drug release is discussed.
\end{abstract}

Key words: Glycerosomes, edge activator, liposomes, glycerol, vesicular system, penetration enhancer, entrapment, encapsulation

In this review article, a new vesicular system with improved entrapment and penetration designed using lipids and glycerol, called glycerosomes is discussed. Glycerosomes has been mainly used in topical preparation so before studying in depth on glycerosomes it is necessary to understand the structure of skin. Skin is made up of two layers, internal and external. The internal layer (deeper layer) is dermis whereas the external layer is epidermis. The external layer is made up of many layers and stratum corneum is present in between these layers ${ }^{[1]}$. It acts as a difficult membrane for drug permeation and is therefore a barrier for most drug substances ${ }^{[1-5]}$. Both the layers differ in thickness. The inner layer thickness varies between 0.3 to $4 \mathrm{~mm}$ consisting of connective tissues and hair follicles whereas the external epidermal layer is of 50 to $150 \mu \mathrm{m}$ thick. It is extended by a hydrolipidic film composed of sweat, sebum and water. Primary function of this film is to protect the skin against microorganisms acting as a protective sheath ${ }^{[2]}$. Topical drug delivery refers to the administration of pharmaceutical dosage forms to

*Address for correspondence E-mail: priyagupta191995@gmail.com

May-June 2020 the skin, eye, nose and vagina for medical purposes ${ }^{[6,7]}$. However, skin is mostly targeted for topical drug delivery ${ }^{[8]}$. Topical drug delivery is advantageous when compared to conventional dosage forms since the former offers good patient compliance and avoids first pass hepatic metabolism ${ }^{[2]}$. Conventional drug delivery refers to classical method of delivering a drug to the body. It has many disadvantages and currently novel drug delivery systems (NDDS) are more preferred. NDDS delivers the drug to the required location and improves drug efficacy ${ }^{[9]}$. Some examples of NDDS include liposomes, transferosomes, ethosomes, nanoparticles and the novel glycerosomes.

Strategies such as iontophoresis ${ }^{[10]}$, sonophoresis, electrophoresis, microneedles and vesicular drug

This is an open access article distributed under the terms of the Creative Commons Attribution-NonCommercial-ShareAlike 3.0 License, which allows others to remix, tweak, and build upon the work non-commercially, as long as the author is credited and the new creations are licensed under the identical terms

Accepted 30 March 2020 Revised 14 February 2020 Received 06 September 2019 Indian J Pharm Sci 2020;82(3):385-397 
delivery systems are used for enhancing topical drug delivery. Among these, vesicular drug delivery seems to be the most favourable and beneficial for topical dug delivery ${ }^{[11]}$. Vesicles are concentric bilayer structures composed of an aqueous core and amphiphiles (lipids and surfactants). These are colloidal particles capable of enclosing both hydrophobic and hydrophilic drugs. Vesicles composed of phospholipids are liposomes and those composed with non-ionic surfactant are niosomes ${ }^{[12,13]}$. Glycerosomes are novel vesicular structures containing phospholipids, water and glycerol in high amounts. Glycerol is harmless, non-toxic and non-irritating in nature and thus safe to use. It is added to vesicular preparations since it results in an increase in fluidity and stability of vesicles. Increased fluidity of glycerosomes aides in better penetration on skin surface than the conventional liposomes. Phospholipids are added to glycerosomes for the formation of bilayer structures. Both synthetic and natural phospholipids can be added to glycerosomes. Cholesterol, stearylamine, diacetyl phosphate are added as stabilizers or for altering the electric charge on vesicles. These novel vesicular structures can be prepared from the methods used for conventional liposomes. Both hydrophilic and lipophilic drugs can be incorporated in glycerosomes. Hydrophilic drugs gets entrapped in aqueous space whereas hydrophobic drugs gets entrapped in phospholipid. Unilamellar or multilamellar vesicles (MLVs) are formed based on the excipients and the method selected for the preparation of glycerosomes. These nanostructures are recently gaining interest worldwide due to their ease of preparation, harmless nature and better characteristics than the conventional vesicular structures ${ }^{[2]}$.

\section{Liposomes:}

Liposomes are spherical bilayer vesicles of phospholipids ${ }^{[14]}$. Although first described by Bangham in $1960^{[15]}$, these were fully understood and accepted in $1970^{[16,17]}$. Hydrophobic tail and hydrophilic head constitutes phospholipids. These phospholipids when brought in contact with water give rise to spherical structures due to the action of acyl chains that are hydrophobic in nature. Apart from being stable thermodynamically, liposome formation is also favoured by various chemical forces namely van der Waals and hydrogen bonding ${ }^{[14,18,19]}$. Being amphiphilic in nature, liposomes can encapsulate both polar and non-polar components ${ }^{[14]}$. Although liposomes have been used for topical and transdermal drug delivery, but it is not considered effective since the liposomes are not able to penetrate deeper into tissues and remain in the superficial tissues ${ }^{[20]}$. These are not only used for topical and transdermal drug delivery but are also used for various other kinds of drug delivery like targeted drug delivery, sustained release. Stability problem of liposomes limits their usage ${ }^{[21,22]}$. There is need for vesicular systems with improved characteristics. Glycerosomes represent such liposomes with improved effects and delivery.

\section{Glycerosomes:}

The concept of glycerosomes was introduced by Manca et al. for the delivery of diclofenac to $\operatorname{skin}^{[23]}$. Glycerosomes are bilayer vesicles used for dermal and transdermal drug delivery. These vesicles differ from conventional liposomes in bilayer fluidity, formed by the addition of phospholipids and varying concentrations of glycerol $(10-30 \% \mathrm{v} / \mathrm{v})^{[23]}$. These are so named, as they contain high amount of glycerol ${ }^{[2]}$. These vesicles deliver the active ingredients to skin with high efficiency ${ }^{[24]}$. Glycerosomes are found to be more stable and possess greater fluidity then liposomes and hence are predominantly used as topical drug delivery systems ${ }^{[23]}$. Glycerol ameliorates the deformability index of liposomal bilayers, thus enhancing skin penetration $^{[23,25]}$.

\section{Glycerosomes composition:}

Glycerosomes are new vesicular systems composed of phospholipids and cholesterol just like conventional liposomes. When dispersed in water, phospholipids quickly assemble themselves as bilayer vesicles ${ }^{[26]}$. Only difference is that in addition to the excipients present in liposomes, these also contain glycerol and water in concentrations of 10, 20, 30, 40 and $50 \%$. Glycerosomes have till now been suggested for topical and skin drug delivery. It is a completely harmless and non-toxic method of drug delivery ${ }^{[23,25,27-30]}$.

\section{Structure of glycerol:}

Glycerol is a viscous liquid and an alcohol. It consists of three hydroxyl groups which renders it hydrophilic properties. Glycerol being a triglyceride can be found in animal fats and vegetable oils. It can be obtained during soap production or as a by-product during the production of biodiesel. It is used in pharmaceutical preparations as a lubricant, humectants, edge activator and emulsifier ${ }^{[31]}$. 


\section{Phospholipids:}

Like conventional liposomes, both natural and synthetic phospholipids can be employed for the preparation of glycerosomes ${ }^{[2]}$. Variety of phospholipids exists because of different hydrophilic head groups, hydrophobic tails and alcohol groups. In addition to being amphiphilic, these are also compatible with almost every ingredient. When dispersed in water, phospholipids assemble in different shapes. Self-assembly in hydration medium depends upon the properties that the phospholipids possess. Different types of phospholipids exist based on their difference in backbone structure and the alcohol moiety that they contain ${ }^{[32]}$.

\section{TYPES OF PHOSPHOLIPIDS}

\section{Glycerophospholipids:}

Glycerophospholipids are obtained from eukaryotes. Glycerol is the main constituent of these lipids. Change in the hydrophilic head group of these lipids results in the formation of cardiolipin, phosphatidylcholine, phosphatidylserine and so on. Whereas change in the acyl chains of these lipids results in the formation of dipalmitoyl phosphatidylcholine and dimyristoyl phosphatidylcholine ${ }^{[32]}$.

\section{Sphingomyelins:}

Obtained from animal cell walls, these phospholipids differ from glycerophospholipids in the fact that these consist of sphingosine backbone whereas the latter consists of glycerol backbone. These not only differ in chemical structures but also differ in the number of groups present in acyl chains. Sphingophospholipids are considered asymmetric and glycerophospholipids are considered symmetric. Acyl groups present in naturally occurring sphingomyelins exceeds 20 whereas the residues of paraffin consist of fewer groups compared to the natural one. Thus it is referred to as asymmetric. Chain length in case of phosphatidylcholine (example of a glycerophospholipids) is equal and thus they are termed as symmetric molecules ${ }^{[32]}$.

\section{Properties that phospholipids to form glycerosomes:}

The phospholipids must be able to form vesicles, which are capable of including pharmaceutical ingredients in them as well as harmless. The phospholipids must give rise to the vesicles that must be degradable in nature. The phospholipids must give rise to the vesicles that must be compatible with other excipients of the formulation.

\section{Cholesterol:}

Animal cell membrane is mostly made up of cholesterol. It is known to affect various properties of membranes. Rigidity, thickness, stability and fluidity of cell membranes depend on cholesterol in one or another way ${ }^{[33]}$. In glycerosomes, the purpose of adding cholesterol is to enhance stability. Cholesterol mainly makes contact with liposomes inner cavity due to its hydrophobic nature, which renders it stable $e^{[34]}$. The amount of cholesterol that can be added in vesicular systems has been reported to be 50 mol percent ${ }^{[21,35-37]}$. Lipid and cholesterol ratio that gives rise to efficient liposomes has been reported to be $2: 1$. However, the reason behind this ratio is not well established. Effect of incorporation of cholesterol in vesicular structures has been widely studied and researchers have concluded that cholesterol plays role in the following ${ }^{[21]}$. It makes the membrane impermeable to water and electrolytes and hence is included in liposome formation ${ }^{[38]}$. It increases the ordering and packing efficiency of lipid bilayers ${ }^{[39]}$. It helps in the deaggregation of lipid vesicles ${ }^{[40]}$. It improves rigidity of lipid bilayers by changing the fluidity of vesicles ${ }^{[41]}$.

\section{Nature of drugs encapsulated:}

Both hydrophilic and lipophilic drugs can be encapsulated in liposomes and glycerosomes ${ }^{[2,42,43]}$. Hydrophilic drugs are entrapped in the aqueous cavity of these vesicular structures whereas the lipophilic moieties are entrapped in the tails of phospholipids. Thin film hydration method is widely used for the encapsulation of hydrophilic moieties. This method although favours the encapsulation of water loving drugs but lowers their encapsulation efficiency ${ }^{[43]}$. Drugs with intermediate $\log \mathrm{P}$ value get entrapped between water soluble and lipid soluble compartments ${ }^{[44,45]}$. These vesicular systems deliver the drug at required site without causing its decomposition.

\section{PROPERTIES OF GLYCEROSOMES, MO- LECULAR GEOMETRY}

Critical packing parameter (CPP) can be defined as the parameter governing the packing of phospholipids together ${ }^{[46]}$. Phospholipids possessing larger CPP form larger liposomes with higher entrapment efficiency ${ }^{[47]}$. Sakai et al. reported that trapping efficiency increases on increasing the unsaturated bonds and bulkiness of the molecule ${ }^{[47]}$. $\mathrm{CPP}=\mathrm{v} / \mathrm{lc} \mathrm{AP}, \mathrm{CPP}=\mathrm{AhP} / \mathrm{AP}$, where $\mathrm{v} / \mathrm{lc}$ is $\mathrm{AhP}, \mathrm{v}$ is the volume of hydrophobic groups, lc is the hydrophobic group length, AP is the cross sectional area 
of hydrophilic head group and Ahp is the cross sectional area of hydrophobic head group. When phospholipids are immersed in water they acquire different shapes namely spherical, inverted, lamellar and cylindrical. These shapes are determined by $\mathrm{CPP}^{[48]}$. Spherical micelles are formed if CPP is equal to or less than $1 / 3$. Cylindrical micelles are formed if CPP is more than $1 / 3$ and less than 1/2. Lamellar structures are formed if CPP becomes equal to 1 . Inverted micelles are formed when value of CPP exceeds a certain value.

\section{METHODS OF PREPARATION GLYCEROSOMES}

Glycerosomes can be prepared using the same methods that are used for the preparation of conventional liposomes ${ }^{[2,23]}$. However, researchers have mostly used thin film hydration method for the preparation of glycerosomes ${ }^{[23,28,49]}$. The various methods of preparation according to the vesicle lamellarity are listed below:

\section{MLVs, lipid hydration method/thin film hydration method:}

In this method, lipid is dissolved in an organic solvent and then dried to form a lipid film. The film so formed is hydrated above transition temperature of lipid by adding aqueous medium. The dispersion is mechanically stirred. If the drug to be encapsulated is hydrophilic, then it is added to aqueous hydration buffer and if the drug to be encapsulated is lipophilic, then it is added to the lipid film ${ }^{[23,50,51]}$. Bangham et al. were the first to describe this method for the formation of MLVs ${ }^{[51,52]}$. The hydration medium can be glycerol in water for preparing glycerosomes ${ }^{[23]}$.

\section{Solvent spherule method:}

In this method phospholipids are mixed with an organic solvent, which is then dissolved in an aqueous medium. The above mixture is then stirred/vortexed for $1 \mathrm{~h}$ under low vacuum to yield lipid spherules with organic solvents (oil in water emulsion). For removal of organic solvents, the spherules are subjected to controlled evaporation on a water bath to yield MLVs ${ }^{[50,51,53]}$.

\section{Small unilamellar vesicles (SUVs) sonication:}

In this method, MLVs are subjected to sonication. The sonication is carried out using either a bath or a probe sonicator. The process is carried out under inert atmosphere. This method suffers from various drawbacks namely ${ }^{[50,51]}$, large molecules are rejected, degradation of compounds take place and encapsulation efficacy reduces.

\section{French pressure cell method:}

In this method, MLVs are made to pass through a small orifice at $4^{\circ}$ and 20000 psi. Although this method produces large liposomes when compared to the ones formed by sonication procedure but suffers from drawbacks like the conditions required for the formation of vesicles are difficult to maintain.

\section{Large unilamellar vesicles (LUVs) reverse phase evaporation method:}

Szoka and Papahadjopoulos were the first to describe this method $^{[54,55]}$. In contrast to spherule method, this results in the formation of water in oil emulsion ${ }^{[50,51,54]}$. The main advantage of reverse phase evaporation method is that it results in the formation of vesicles with large aqueous to lipid ratio, entrapping more of the aqueous medium ${ }^{[54,55]}$. The procedure comprises of adding the lipids to a $50-\mathrm{ml}$ round bottom flask. The lipids can be added alone or mixed with other lipids such as cholesterol. Solvent is removed by controlled evaporation. After this step, the dried lipid film is purged with nitrogen gas and redissolved in an organic solvent. This organic solvent is the one in which inverted micelles formation takes place. Hydration medium is added at this step under nitrogen atmosphere. The obtained two phase system is sonicated in a bath sonicator for about 2-5 min until a clear one phase dispersion or homogenous opalescent dispersion is formed. The dispersion is then added to a rotary evaporator for removal of organic solvent at stated conditions i.e. reduced pressure, $20-25^{\circ}$ and $200 \mathrm{rpm}$. The mixture starts to froth during evaporation and results in viscous gel that gets converted to aqueous suspension $^{[51,54,55]}$. Again, aqueous buffer is added and the suspension is subjected to evaporation for nearly $15 \mathrm{~min}$ at $20^{\circ[46]}$. The resultant product is then dialysed and passed through a Sepharose 4B column or it can be centrifuged. Centrifugation or passage through a column is required for removing nonencapsulated material[ ${ }^{[55]}$.

\section{Detergent removal method/detergent dialysis method:}

This is a widely used method for the encapsulation of protein and biological molecules. Non-ionic, anionic and cationic detergents can be used for the formation of vesicles. In this method, detergents possessing high critical micelle concentration are utilized. Phospholipids are dissolved in detergents and after that removal 
of detergents is carried out. Gel chromatography or Lipoprep can be used for the separation of detergents. Advantages include formation of homogenous vesicles with efficient replication ${ }^{[50,51,54]}$. Alpes et al. investigated the role of alkyl maltosides as detergents for dialysis method of liposomes preparation. It was reported that detergent removal speed depends mainly on permeation of dialysis membrane and on the critical micelle concentration of detergent. It was also observed that, alkyl chain length majorly governs the critical micelle concentration. It was reported that decyl maltoside is the ideal detergent for the preparation of vesicles by detergent removal method due to its speed of removal in the formation of vesicles ${ }^{[56]}$.

\section{Double emulsion evaporation:}

In this method, double emulsion of the type $\mathrm{W} 1 / \mathrm{O} / \mathrm{W} 2$ is prepared. It consists of inner and outer aqueous phase ${ }^{[54]}$. Outer phase consists of dispersed individual oil globules whereas inner aqueous phase occurs as small droplets in each oil globule of outer aqueous phase ${ }^{[57]}$. The process of double emulsion evaporation comprises of following steps ${ }^{[54,58-60]}$. Aqueous phase consisting of the drug is dissolved in water and added to organic solvent containing lipids. This forms water in oil emulsion which is then properly homogenized to form primary emulsion (W1/O). Primary emulsion when combined with outer aqueous phase containing stabilizer gets converted to double emulsion.

\section{Solvent injections method, ether injection method:}

Watkins described this method ${ }^{[61]}$, in which lipids are dissolved in diethyl ether/ether methanol mixture. These are then injected to a warm aqueous solution that contains the material to be encapsulated. The warm aqueous phase should be maintained above the boiling point of ether. Usually $55-65^{\circ}$ temperature is maintained during encapsulation ${ }^{[50,54]}$. The speed of injection should be slow and not rapid. On coming in contact with warm aqueous phase, ether evaporates giving rise to unilamellar vesicles ${ }^{[31,61]}$. Disadvantages of this method include low yield of heterogenous liposomes, along with the exposure of compounds which are to be encapsulated to high temperature and organic solvents ${ }^{[62]}$.

\section{Ethanol injection method:}

Ethanol injection method was reported by Batzri and Korn in $1976^{[63,64]}$. In this method, lipid is dissolved in ethanol and made to pass through a small orifice that may be a syringe in excess aqueous medium. The speed of injecting ethanolic lipid solution in aqueous medium should be such that complete mixing of the two is attained. Complete mixing of the two is necessary for immediate dilution of ethanol in hydration medium and for the dispersion of phospholipids in water ${ }^{[63,64]}$. Main advantage of this method lies in the fact that without sonication and extrusion one can obtain small liposomes under the size range of $100 \mathrm{~nm}$ simply by injecting lipid solution dissolved in ether in water ${ }^{[62]}$. Also, homogenous and dilute liposomes are obtained ${ }^{[64]}$. Disadvantage of ethanol injection method includes the constraint due to lipid solubility in ethanol, volume of ethanol that can be added to aqueous medium is limited and thus the lipid which can be added to ethanol is limited. Ethanol remains in liposomes but can be removed by dialysis ${ }^{[64]}$.

\section{Calcium-induced fusion method:}

In this method, LUVs are formed by the addition of calcium $\left(\mathrm{Ca}^{2+}\right)$ to SUVs which results in fusion. Fusion gives rise to large planar lamellae which transform to cochleate cylinders. These are then mixed with EDTA

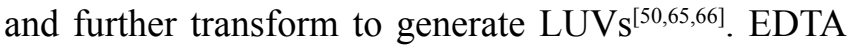
helps in re-establishing negative charge and maintains the fluidity of membrane ${ }^{[65]}$.

\section{Microfluidization method:}

This method utilises a microfluidizer capable of producing liposomes continuously in large amount with high aqueous volume. Mayhew et al. ${ }^{[67]}$ prepared liposomes using microfluidizer and suggested that the prepared liposomes resembled same properties as that of small extruded multilamellar liposomes. On comparison with normal multilamellar liposomes, the ones prepared using microfluidizer yielded smaller liposomes with uniform size distribution ${ }^{[67]}$. The process as described in Mayhew et al. involved addition of aqueous lipid suspensions to a reservoir. From the reservoir the suspension passes onto pump and through filters fluid is pumped. Fluid must be pumped at high pressure and through $5 \mu \mathrm{m}$ filter size. After that it passes to interaction chamber, here the streams interface in micro channels at exorbitant speed after being separated into two different streams. Outflow is processed and the liposomes are analysed accordingly ${ }^{[67]}$.

\section{Freeze-thaw method:}

This method makes use of freezing and thawing procedure $^{[50,68,69]}$. Freeze-thaw method can be used only 
with mixtures of charged phospholipids i.e. the ones exhibiting positive and negative charge or this method can be applied to the phospholipids, which are crude in nature ${ }^{[69]}$. Small unilamellar liposomes are speedily frozen followed by thawing. They are then sonicated to give LUVs. This procedure results in the formation of LUVs due to the fusion of bilayers of SUVs upon freezing flowed by thawing. Increased concentration of liposomes or increased ionic strength reduces the formation of liposomes by this method ${ }^{[50,68,69]}$.

\section{CRITICAL PARAMETERS GLYCEROSOMES}

\section{Particle size analysis:}

Particle size analysis and polydispersity index (PI) of glycerosomepreparation can be tested on azetasizer. This process is known as dynamic laser light scattering ${ }^{[27,30]}$ or photon correlation spectroscopy ${ }^{[22,23,28,49]}$. Usually a Malvern zetasizer is used for this purpose $\mathrm{e}^{[22,23,28,49]}$.

\section{Vesicle formation:}

Vesicle formation is confirmed by the use of SEM (scanning electron microscopy), TEM (transmission electron microscopy) and cryo-TEM ${ }^{[22,27]}$. In TEM, first the samples are stained with $1 \%$ phosphotungstic acid and then analysed using an electronic microscope. Cryo-TEM consists of coating the samples on a carbon rod and dipping it in ethane at its melting point. These are then analysed under the TEM.

\section{Determination of deformation index:}

In this, the glycerosomal preparations are made to pass through a membrane extruder, which exhibits a specific pore size and this is done at a specific pressure using an extruder. The pore size of the membrane should be smaller than the mean size of phospholipid vesicles. For penetration through skin, flexible vesicles are required to easily pass through skin pores. Glycerol is supposed to form this type of vesicles and thus deformation index is calculated for ascertaining whether glycerol can form vesicles that can change their shape ${ }^{[23]}$.

\section{Determination of entrapment efficiency (\%):}

Different techniques are used for calculating entrapment efficiency. Manca et al. ${ }^{[22,23,27,28]}$ have used the method of dialysis. They passed the glycerosome formulation from dialysis tube of 12000 to 14000 Dalton and then separated entrapped formulations from the non-entrapped one. These were then assayed for drug content. Entrapment is measured on the basis of percentage of drug which was obtained after dialysis to that which was taken before dialysis, formula $=$ obtained drug content/initial drug content $\times 100$.

\section{Determination of penetration:}

This evaluation parameter helps in determining the extent to which glycerosomes has penetrated the skin layer. This can be performed by ex vivo means. It helps in determining drug delivery through the skin layer. Franz diffusion cell is used for this purpose. Animal skin is mounted between the donor and the receptor compartments, such that the stratum corneum of skin is towards the donor side. After the glycerosome preparations are applied to the stratum corneum at definite intervals, the medium collected in the receptor compartment is taken out and replaced by fresh medium. It is analysed for drug content using a suitable method (UV or HPLC). When all the samples have been administered then the skin is detached from Franz diffusion cell. After separating epidermis from dermis, the layers are then sonicated and the amount of drug determined ${ }^{[22,23,28,49]}$.

\section{Determination of drug release in vitro:}

Dialysis bag is used for determining the in vitro drug release from glycerosomes. In this procedure, $1 \mathrm{ml}$ of glycerosome preparation is added to dialysis bag and the bag is immersed in phosphate buffer. Samples are taken out at definite intervals and analysed using a UV or a HPLC method ${ }^{[49]}$.

\section{Determination of fluidity:}

Differential scanning colorimetry (DSC) studies are used for determining the fluidity of lipid bilayers. This method helps one in determining the transition temperature of phospholipids used. It has been reported that transition temperature of phospholipids indicates its interaction with external substances. Thus on glycerol addition, change in transition temperature is observed that further leads to the conclusion that the glycerosomes possess more fluidity. Glycerol causes reduction in transition temperature of phospholipids due to the hydration effect that it has on lipid bilayers which in turn causes reduction in the melting point of acyl chain of phospholipids. Cholesterol and amphipathic drug used also impact transition temperature of the phospholipid. Cholesterol disturbs the bilayer packing by preventing the growth of pretransition peak over main transition peak. Manca et al. reported the effect of amphiphilic diclofenac sodium. It was reported 
that diclofenac enhanced glycerol interaction with phospholipid bilayers as being amphipathic in nature and gets inserted in lipid bilayers and thus causes a reduction in transition temperature ${ }^{[23,28]}$. Salem et al. reported the topical delivery of celecoxib and cupferron by formulating glycerosomes and its ge ${ }^{[49]}$. They used soybean phosphatidyl choline as the phospholipid. They showed the effect on fluidity of vesicles by comparing the transition temperatures of celecoxib and cupferron and unlike Manconi et al. ${ }^{[23]}$ who reported the effect on vesicles by relating phospholipids Tm before and after formulating them in glycerosomes. Salem et al. compared the DSC peaks of pure celecoxib and cupferron and noticed pointed peaks at 162 and $177^{\circ}$, whereas, glycerosomal formulations of celecoxib and cupferron gave peaks at somewhat lower temperature namely 145 and $164^{\circ}$. This showed better encapsulation efficiency and more fluidity of vesicles ${ }^{[49]}$.

\section{Determination of stability:}

Determining zeta potential using a Zetasizer helps in determining the charges present on the surface of glycerosomes that may be present due to the addition of charged species in formulation and also tells about the stability of the preparation. Negative zeta potential results in increased stability of the formulation. It has been reported that glycerosomes exhibit better stability than the liposomes. Researchers have reported that throughout the period of stability studies, glycerosomes did not show any change in vesicle size and $\mathrm{PI}^{[22,23,28]}$. However, some researchers observed change in vesicle size of glycerosomes. Manca et al. reported the formation of curcumin glycerosomes and curcumin polymer glycerosomes ${ }^{[27]}$. Stability studies were conducted by storing the above formulations for $90 \mathrm{~d}$ at room temperature. The results indicated that glycerosomes became larger in size. This change was evident after $30 \mathrm{~d}$ and then there was no change until the stability studies were completed. Hyaluronan containing glycerosomes showed increase in size after $60 \mathrm{~d}$ whereas trimethyl chitosan showed no change in their size ${ }^{[27]}$. Zhang et al. had reported the formulation of paeniflorin glycerosomes containing essential oil of Speranskia tuberculata for improving skin delivery ${ }^{[25]}$. Stability studies were carried out by storing the oil containing glycerosomes at temperatures as low as $4^{\circ}$ and at normal temperatures. Formulations were analysed on specific days. Results indicated that the glycerosomes stored at room temperature showed difference in particle size. The particle size increased, whereas the glycerosomes which were stored at $4^{\circ}$ showed no change in particle size ${ }^{[25]}$. Manca et al. had speculated that glycerol acted as a non-aggregating agent because glycerosomes showed no change in their size at the end of stability studies while liposomes size was changed $^{[28]}$.

\section{APPLICATIONS OF GLYCEROSOMES}

\section{Topical delivery with glycerosomes:}

Manca et al. reported formulating glycerosomes containing diclofenac sodium for transdermal drug delivery ${ }^{[28]}$ They hydrated dipalmitoylglycerophosphatidylcholine and cholesterol films with aqueous glycerol solution (10-30\% v/v) and compared them with conventional liposomes. Results indicated that glycerosomes and liposomes were in the size range of 81 and $97 \mathrm{~nm}$, oligo/multilamellar and spherical vesicles were formed. Glycerol acted as edge activator when used in concentrations more than $10 \%$ as shown by defomability index, DSC studies indicated the more fluidity state of glycerosomes than the conventional liposomes and in vitro transdermal studies showed the improved permeation of diclofenac. The penetration studies were carried out using pig skin mounted on a Franz diffusion cell. The permeation efficiency of glycerosomes, liposomes and a commercial gel preparation were evaluated. Liposomes and commercial gel served as controls. The vesicular preparations were added to the donor compartment and after fixed time intervals of 2, 4, 6 and $8 \mathrm{~h}$, samples were withdrawn and drug content was analysed. Drug content in the stratum corneum layer was analysed. Cumulative amount of drug permeated for $8 \mathrm{~h}$ was determined for glycerosomes as well as for control preparations. Flux (J) was calculated utilizing the slope of the plot. It was then observed that, diclofenac permeated to a higher extent when delivered from glycerosomes rather than from liposome and commercial gel preparation. It was also reported that $30 \%$ glycerosomes resulted in maximum transdermal drug delivery ${ }^{[23]}$. Manca et al. reported the entrapment of quercetin in glycerosomes to improve skin penetration and bioavailability ${ }^{[22]}$. Quercetin possesses high antioxidant activity but its skin permeability is low. Results indicated unilamellar vesicles with size range of $80-110 \mathrm{~nm}$. Small angle X-ray scattering (SAXS) studies indicated that glycerol could change the bilayer packing by interacting with polar portions of phospholipids. The formed glycerosomes were compared to liposomes. It was reported that on increasing the concentration of glycerol, its accumulation in skin layer increases as 
evident by in vitro studies. Both vesicles were evaluated for physicochemical properties, antioxidant activity and transdermal delivery. Both liposomes as well as glycerosomes were shown to exhibit unilamellar structure as given by Cryo-TEM. Photon correlation spectroscopy indicated the homogeneity and particle size of glycerosomes. It was reported that 50 and $30 \%$ empty glycerosomes displayed $116 \mathrm{~nm}$ and $134 \mathrm{~nm}$ size, respectively. Also, somewhat broader size distribution was exhibited by the unpurified vesicles due to the possible reason of presence of unentrapped drug in them. Both drug-loaded vesicles (liposomes and $30 \%$ glycerosomes) exhibited larger particle size. Negative zeta potential was shown by both the vesicular formulations indicating good stability. It was reported that with increasing glycerol concentration, entrapment efficiency decreased due to the possible reason of increased drug solubility in vesicular membranes. Size distribution was evaluated after $90 \mathrm{~d}$ for testing the stability of vesicular membranes. It was observed that while liposomes increased their size in $30 \mathrm{~d}$, glycerosomes showed no change in their size, which clearly highlighted the role of glycerol as a vesicular stabilizer. SAXS studies were performed to evaluate the structure of vesicles formed and for determining the role of glycerol and drug on selfassembly of amphiphile. Intense and widened peaks were observed for empty and drug-loaded 40-50\% glycerosomes. It was concluded that addition of drug did not affect bilayer arrangement and SAXS pattern. Glycerol at low concentrations increased amplitude of phospholipids by interacting with polar head groups whereas at high concentrations (40 and $50 \%$ ), glycerol reduced the amplitude. In vitro studies carried out using new-born pig skin demonstrated the high efficiency of glycerosomes accumulation in different skin layers. It was reported that 30-50 \% glycerol vesicles accumulated the most in skin and there occurred no permeation to the receptor compartment during the experimental studies. It was also noted that, liposomes and glycerosomes containing less glycerol showed low drug deposition in skin. Confocal scanning microscopy results were similar to the results obtained in the in vitro studies. For confocal studies, formulations were tagged by fluorescent phospholipid Rho-Pe and in vitro permeation studies were performed. Skin sections were cut after completion of the studies. It was observed that $40 \%$ glycerosomes have accumulated to the same extent as $50 \%$ glycerosomes and $10-20 \%$ glycerosome distribution pattern was similar to liposomes and they were distributed to the external layers of skin and not to the deeper ones. Thirty percent Rho-Pe glycerosomes were distributed in the stratum corneum for $1 \mathrm{~h}$ but after $4 \mathrm{~h}$ they were confined to epidermis. The best results were exhibited by 40 and $50 \%$ glycerosomes. It was observed that fluorescence for both the glycerosomes was detected in stratum corneum and epidermis at $1 \mathrm{~h}$ and this became even more prominent at $4 \mathrm{~h}$. Antioxidant studies of quercetin liposomes and glycerosomes were carried out and compared with free drug solution. DPPH assay method was employed for quantification. Glycerosomes and quercetin solution exhibited $\sim 95$ $\pm 1 \%$, and liposomes exhibited $\sim 87 \pm 2 \%$ free radical scavenging activity. The radical scavenging activity of quercetin wasn't affected by its encapsulation in vesicular forms. Oxidative stress damage studies were performed in keratinocytes. The cells were stressed by hydrogen peroxide that resulted in decreased viability up to $26 \%$. It was observed that quercetin in vesicle form increased the viability to $70 \%$ whereas quercetin drug solution was able to increase the viability to only $48 \%$. Uptake of quercetin vesicle formulations by keratinocytes was also studied. These were marked with Rho-Pe and incubated with cells. Confocal scanning microscope was used and the study was carried out at $2 \mathrm{~h}$ and $4 \mathrm{~h}$. Green colour was used for glycerosomes while red was used for denoting phospholipids. Results indicated that cells treated with liposomes and 10-20\% glycerosomes exhibited green fluorescence in first $2 \mathrm{~h}$ whereas both red and green fluorescence were visible in $4 \mathrm{~h}$. For cells treated with $30-50 \%$ glycerosomes both red and green fluorescence was visible from starting of the experiment. Endocytosis was considered as the mechanism behind transfer of drug and phospholipid in cells ${ }^{[22]}$.

Manca et al. reported the formulation of glycerosomes using a cheap phospholipid for increasing their permeability through skin. They used a mixture of hydrogenated soy phosphatidylcholine $(\mathrm{P} 90 \mathrm{H})^{[28]}$. Diclofenac salt was encapsulated in glycerosomes and glycerol was utilized in 10, 20 and $30 \%$ in aqueous medium. The vesicles were fully characterized. Results indicated the vesicle size to be of $131 \mathrm{~nm}$, larger than the ones reported by Manca et al. using DPPC as phospholipid ${ }^{[23,28]}$.

Rani et al. had reported the formulation of minoxidil glycerosomes for topical drug delivery ${ }^{[30]}$. Lipid thin film hydration method was utilized for the preparation of glycerosomes. Results indicated higher entrapment efficiency, improved surface area of formed glycerosomes and increased cumulative drug 
release. They utilized egg membrane and mounted it on Franz diffusion cell. Nine glycerosomes preparation (2 $\mathrm{mg}$ ) were added to the donor chamber. Samples were withdrawn at definite time intervals and were analysed using UV spectroscopy. The results predicted highest cumulative drug release at $16 \mathrm{~h}^{[30]}$. Abdellatif et al. reported formulating nanovesicular systems of sertaconazole nitrate for treating skin fungal infection ${ }^{[70]}$. In vitro, in vivo and ex vivo evaluations were carried out. Liposomes, glycerosomes, transferosomes and ethosomes were formulated and characterized. The optimized vesicular system was chosen and was further converted into a gel. Confocal laser scanning microscopy was used in permeation studies. For checking the antifungal efficiency of the gel in vivo studies were carried out by comparing it with commercially available cream. The results indicated that transferosomal gel exhibited more flux when compared to commercial gel due to the addition of surfactant as edge activator. Glycerosomes, exhibited high entrapment efficiency and smallest particle size after transferosomes. This was due to the presence of glycerol. Glycerol modified lipid bilayers that resulted in small size of vesicles and more entrapment. Ex vivo skin penetration results also displayed the high penetration efficacy of glycerosomes and transferosome rather than the conventional liposomes. The reason assigned by authors was the elastic nature of former vesicles by which the vesicles were able to squeeze themselves deeper into skin layer rather than liposomes. They reported that, glycerol modified bilayers of lipid that resulted in reduction of skin barrier and consequently more drug was penetrated ${ }^{[70]}$.

Vitonyte et al. had reported the formation of glycerosomes and penetration enhancer vesicles using resveratrol and gallic acid for safeguarding the skin against oxidation and microbial attacks ${ }^{[71]}$. These compounds were chosen due to their antioxidant and antibacterial nature and also since these have poor bioavailability when applied to skin. Resveratrol-gallic acid liposomes, resveratrol-gallic acid penetration enhancer-containing vesicles (PEVs), resveratrolgallic acid glycerosomes and empty liposomes were prepared, among which PEVs and glycerosomes were the reference. Glycerosomes and PEGylated PEVs (PG-PEVs) being more viscous showed better retention on the skin surface as compared to the conventional liposomes. In vitro skin penetration studies were carried out using pig skin in Franz diffusion cell. Different formulations were applied on the surface of pig skin mounted on the cell. After the end of study, stratum corneum was separated from the skin. The samples were analysed for drug deposition in skin layers as well as biological fluid present in Franz diffusion cell. Results showed that, glycerol aided in increased deposition of resveratrol and gallic acid. This was due to the nature of glycerol that modified lipid packing present in stratum corneum which resulted in increased driving force for diffusion of drugs ${ }^{[71]}$.

Manca et al. reported the formulation of glycerosomes and liposomes using 1,2-dimyristoyl-sn-glycero3-phosphatidycholine phospholipid and diclofenac sodium as the drug. Results indicated that glycerosomes were able to enhance the fluidity and performance when applied to the skin surface in comparison to liposomes $^{[72]}$.

Salem et al. reported the formulation of celecoxib and cupferron glycerosomes and converted the formulation to gel for topical drug delivery. They evaluated glycerosomes formulation for antiinflammatory effect. Cupferron was chosen, so as to eliminate or reduce the side effects of celecoxib which is a cox- 2 inhibitor. Thin film hydration method was used for the preparation of glycerosome and vesicles were fully characterized. Evaluation tests for gel were also performed. Results depicted high cumulative drug penetration of glycerol vesicles and antiinflammatory action on rat paw edema. In vitro drug release studies were performed using a dialysis bag. One millilitre of glycerosome formulation of each drug was added to dialysis bag which was then immersed in phosphate buffer saline. It was observed that glycerosomes of celecoxib and cupferron exhibited greater drug release than the glycerosome gels. It was also observed that increasing the concentration of glycerol led to decrease in the drug release from vesicles. Ex vivo permeation studies using shaved rat skin was carried out in Franz diffusion cells. Permeation studies depicted increased permeability of celecoxib and cupferron formulations which was attributed to the presence of phospholipid and Tween 80 concentration. Out of the 17 formulations tested for both the drugs, formulation 2 of celecoxib and formulation 1 of cupferron showed highest penetration. It was assumed that phospholipid caused hydration of skin and Tween 80 provided the deformability required to the vesicles. In vivo histopathological studies, carried out on drug-loaded glycerosome gels displayed prominent antiinflammatory action on rat paw oedema, indicative of enhanced bioavailability of both the drugs ${ }^{[49]}$. 


\section{Inhaled drug delivery using glycerosomes:}

Manca et al. reported the incorporation of curcumin in polymer glycerosomes for delivering curcumin to lungs. They formulated glycerosomes and polymer glycerosomes, combined the later with two polymers for preparing polymer glycerosomes. Results indicated that glycerosomes and polymer glycerosomes were unilamellar and of 65-112 $\mathrm{nm}$ size range. Also, curcumin accumulation to lungs was indicated from both the formulations, but polymer glycerosomes exhibited greater biocompatibility in vitro. Researchers concluded that hydrophilic polymers and glycerol resulted in the formation of network rather than a coating around polymer glycerosomes. This mesh/ network aids in efficient delivery of phospholipid vesicles in vivo by making small changes in dispersion medium and forming stable vesicles than before. Also, polymers offer several other advantages like safe to use and are biocompatible. These also possess high delivery properties. Polymer glycerosomes resulted in effective lung delivery due to their aerosolization, which also helped in improving the antiinflammatory and antioxidant activity of curcumin. All in all, polymer glycerosomes were reported to be suitable for lung delivery and are expected to improve patience compliance and avoid hepatic first pass metabolism ${ }^{[27]}$.

Melis et al. reported the formulation of polymer glycerosomes for lung delivery to treat pulmonary diseases. These were loaded with rifampicin. Polymer glycerosomes and glycerosomes without polymer were prepared. Trimethyl chitosan chloride and sodium hyaluronate was added for preparing polymer glycerosomes. Results indicated that polymer glycerosomes led to increase in drug concentration in pulmonary system. Polymers resulted in the formation of stable vesicles that led to more deposition of drug in lungs, an increase in mean diameter of vesicles, these were aerosolized easily and were delivered to the farthest extent using a next generation impactor ${ }^{[73]}$.

\section{Oral delivery with glycerosomes:}

Manconi et al. reported the formulation of liposomes, glycerosomes and PEGylated penetration enhancing vesicles for the delivery of extract of citrus lemon to oral cavity. The extract of phenol was prepared by maceration of the fruit rind with ethanol. This extract was then loaded in different vesicular systems. These were prepared for enhancing the efficacy of treatment through vesicular systems for diseases of oral cavity. It was stated that glycerosomes and PG-PEVs were better than the conventional liposomes since these vesicles possessed enhanced penetration. PI and zeta potential were evaluated for both extract-loaded and empty vesicles. It was reported that empty liposomes, glycerosomes and PEGylayed penetration enhancing vesicles displayed small size. It was also observed that increasing amount of glycerol (25 and $50 \%$ ) and glycol (12, 25 and $50 \%)$ led to an increase in vesicles size approximating to $100 \mathrm{~nm}$. The reason attributed to increased size of vesicles with high concentration of glycerol and glycol was, the solvents ability to modify bilayer assembly of lipids and thereby favouring the formation of vesicles with increased size. All extract loaded vesicles were reported to be large in size. Empty vesicles displayed negative zeta potential whereas loaded vesicles zeta potential were less negative. Size distribution was narrow for extract loaded glycerosomes $(\mathrm{PI}<0.25)$ and PEGylated penetration vesicles displayed a polydispersed size distribution $(\mathrm{PI}<0.39)$. Entrapment efficiency of all the vesicles irrespective of their type was approximately $63 \%$. Glycerosomes and PEGylated penetration vesicles showed good stability due to their viscous nature unlike the conventional liposomes. The glycols imparted viscosity to vesicles, which in turn resulted in preventing fusion. Antioxidant as well as antibacterial activities of vesicles were also evaluated. For antioxidant activity, keratinocytes were used since they are from epithelium of oral mucosa. The cells were stressed with hydrogen peroxide and extract dispersed in water was chosen as reference. It was reported that glycerosomes and PEGylated penetration vesicles led to an increase in viability up to $100 \%$ and liposomes increased the viability to $90 \%$. Antibacterial activity was tested by measuring the zone of inhibition of bacterial growth. Extract dispersed in water was chosen as reference. It was reported that all the vesicles inhibited the bacterial growth to the same extent. Moreover it was stated that entrapment of extract in vesicles and delivering it to oral cavity may help in efficient distribution of contents along with an increase in the release of contents in oral mucosa ${ }^{[74]}$.

\section{GLYCEROSOME-BASED DRUG DELIVERY NANOCARRIERS}

Polymeric glycerosomes are prepared by adding a suitable polymer to the glycerosome preparation. Polymer addition was during the hydration step. This led to the formulation of polymer-coated glycerosomes. These vesicles internally form a mesh of polymers with improvement of the in vivo characteristics of the system $^{[27,73]}$. Key features of polymeric glycerosomes 
TABLE 1: GRANTED PATENT OF GLYCEROSOMES

\begin{tabular}{|c|c|c|c|c|c|c|}
\hline Title & $\begin{array}{c}\text { Patent } \\
\text { Number }\end{array}$ & Type & Filed & $\begin{array}{l}\text { Date of } \\
\text { Patent }\end{array}$ & Assignee & Inventors \\
\hline $\begin{array}{l}\text { Glycerosomes and } \\
\text { use thereof in } \\
\text { pharmaceutical and } \\
\text { cosmetic preparations } \\
\text { for topical application }\end{array}$ & 8778367 & Grant & $\begin{array}{c}\text { March 8, } \\
2010\end{array}$ & $\begin{array}{c}\text { July } 15, \\
2014\end{array}$ & $\begin{array}{l}\text { PRIGEN } \\
\text { S.R.L. }\end{array}$ & $\begin{array}{c}\text { Marco Zaru, Maria Letizia } \\
\text { Manca, } \\
\text { Anna Maria Fadda, } \\
\text { Gaetano Orsini }\end{array}$ \\
\hline
\end{tabular}

included, enhanced biocompatibilities, improved drug deposition at the target site, avoidance of first pass metabolism and delivery of the contents at the required location of lungs, safety and nontoxic in nature. These formulations can easily be converted to spray form and thus aids in pulmonary drug delivery.

\section{ADVANTAGES OF GLYCEROSOMES}

This new vesicular system is a harmless and nontoxic topical drug delivery system. Glycerosomes do not depend on transition temperatures for their formation. They can be formed at room temperature (30 or $25^{\circ}$ ) unlike conventional liposomes. They improve penetration of drug in stratum corneum and deliver it to the inner layers of skin by acting as edge activator and penetration enhancer. These vesicles are with improved entrapment, fluidity and stability. Stability is improved by changing fluidity of lipid bilayer and by forming viscous preparations. Also, forms deformable and flexible vesicles. Glycerol, being viscous in nature homogenously spreads on skin and also there occurs no leakage of active pharmaceutical ingredient unlike conventional liposomes ${ }^{[68]}$. Glycerosomes exhibit the property of changing the configuration in which hydrophilic chains of phospholipids are arranged and they can change the way in which other vesicles of the system interact with each other. This is possible since the dielectric constant of system can change in glycerosomes ${ }^{[71]}$. Glycerosomes also change the plasticity of the skin layer and improve it. These increase the water content in the stratum corneum and minimise the obstacles in transdermal drug delivery ${ }^{[23]}$. These vesicles are unique in the sense that these can act as penetration enhancing vesicles as well as the elastic ones $^{[23,75]}$.

\section{DISADVANTAGES OF GLYCEROSOMES}

Glycerosomes formed from less than $20 \%$ of glycerol are less productive than those formed from $20 \%$ or more glycerol concentration. Vesicles of less than 20 $\%$ glycerol possess low viscosity, reduced penetration and low flexibility. Another disadvantage includes the disturbance in orientation of phospholipids. Vesicles exhibiting high transition temperatures results in disorientation at room temperature ${ }^{[2]}$. Increased entrapment of glycerol in vesicles leads to delayed drug release as glycerol disturbs the osmotic balance between receptor and donor $\operatorname{sides}^{[49]}$. Addition of glycerol to vesicles leads to increased particle size and reduced drug release ${ }^{[25,49]}$. Glycerosome viscosity on one hand results in improved stability but on other hand it may prolong the time for vesicles to reach the skin surface from the formulation ${ }^{[71]}$. Granted patents of glycerosomes ${ }^{[2]}$ have been listed in Table 1.

In conclusion, glycerosomes represent new vesicular systems composed of glycerol in varying proportions. These systems are capable of avoiding skin barrier layers and delivering the drug to deeper skin tissues. Primarily used for topical drug delivery due to its ability of enhancing penetration through skin, increasing entrapment of drug in vesicles, forms flexible and more fluidic vesicles. Glycerol acts as a modifier for the skin layers by changing the arrangement of skin layers. This system has been mostly used for transdermal drug delivery but Manca et al. prepared glycerosomes of curcumin for lung delivery ${ }^{[2]}$. These emerging vesicles can easily be formed at room temperature and do not require attainment of transition temperature of phospholipids. Entrapment of more amount of drug sometimes results in delayed drug release due to osmotic imbalance on both sides but these preparations have proven themselves in the past few years in terms of stability, fluidity, entrapment and viscosity. These vesicles can also be used for ocular drug delivery in near future, since these will be helpful for delivering those drugs which have poor corneal penetration and low entrapment in liposomes.

\section{Acknowledgement:}

The authors would like to convey thanks to NIET (Pharmacy Institute, Greater Noida) for providing the infrastructure for carrying out the present study.

\section{Conflicts of interest:}

There are no conflicts of interest among the authors. 


\section{REFERENCES}

1. Cristiano MC, Felisa C, Maria C, Donatella P. Chapter 4 -Innovative vesicles for dermal and transdermal drug delivery. In: Grumezescu Alexandru Mihai. Lipid nanocarriers for drug targeting. William Andrew 2018;pp:175-97.

2. Zaru M, Manca ML, Fadda AM, Orsini G. Glycerosomes and use thereof in pharmaceutical and cosmetic preparations for topical applications. USA Patent 2014;8:778,367 B2.

3. Kumar B, Kumar V, Rana V, Jain S, Tiwary AK. Thermotropic and spectroscopic behavior of skin: relationship with percutaneous permeation enhancement. Curr Drug Deliv 2006;3:95-113.

4. Bommannan D, Potts RO, Guy RH. Examination of stratum corneum barrier function in vivo by infrared spectroscopy. J Invest Dermatol 1990;95:403-8.

5. Sr LPG, Hill James M. Modern iontophoresis for local drug delivery. Int J Pharm 1995;123:159-71.

6. Hadgraft J, Lane ME. Advanced topical formulations (ATF). Int J Pharm 2016;514:52-7.

7. Malik, DS, Mital N, Kaur G. Topical drug delivery systems: a patent review. Expert Opin Ther Pat 2016;26:213-28.

8. Bhowmik D, Gopinath H, Kumar BP, Duraivel S, Kumar KPS. Recent advances in novel topical drug delivery system. Pharm Innov 2012;1:1-12.

9. Yadav D, Kumar S, Pandey D, Dutta RK. Liposomes for drug delivery. J Biotechnol Biomater 2017;7:1-8.

10. Dhote V, Bhatnagar P, Mishra PK, Mahajan SC, Mishra DK. Iontophoresis: a potential emergence of a transdermal drug delivery system. Sci Pharm 2012;80:1-28.

11. Kumar R, Singh M, Bala R, Seth N, Rana AC. Transferosomes: A novel approach for transdermal drug delivery. Int Res J Pharm 2012;3:20-24.

12. Kumar GP, Rajeshwarrao P. Nonionic surfactant vesicular systems for effective drug delivery - an overview. Acta Pharm Sin B 2011;1:208-219.

13. Kaushik AY. Transferosome: The drug loaded ultradeformable vesicles for transdermal drug delivery. Int Res $\mathrm{J}$ Pharm 2011;2:40-2.

14. Bhushan PS, Vladimir CV, Vladimir TP. New developments in liposomal drug delivery. Chem Rev 2015;115:10938-66.

15. Bangham AD, Standish MM, Watkins JC. Diffusion of univalent ions across the lamellae of swollen phospholipids. J Mol Biol 1965;13:238-52.

16. Sessa G, Weissmann G. Incorporation of lysozyme into liposomes. a model for structure-linked latency. J Biol Chem 1970;245:3295-301.

17. Gregoriadis G, The carrier potential of liposomes in biology and medicine (first of two parts). N Engl J Med 1976;295:70410.

18. Israelachvili JN, Marcelja S, Horn RG. Physical principles of membrane organization. Q Rev Biophys 1980;13:121-200.

19. Lasic DD, Novel applications of liposomes. Trends Biotechnol 1998;16:307-321.

20. Choi MJ, Maibach HI, Liposomes and niosomes as topical drug delivery systems. Skin Pharmacol Physiol 2005;18:209-219.

21. Briuglia ML, Rotella C, McFarlane A, Lamprou DA. Influence of cholesterol on liposome stability and on in vitro drug release. Drug Deliv and Transl Res 2015;5:231-42.

22. Manca ML, Castangiaa I, Caddeoa C, Pando D, Escribano E, Valenti D, et al. Improvement of quercetin protective effect against oxidative stress skin damages by incorporation in nanovesicles. Colloids Surf B Biointerfaces 2014;123:566-74.

23. Manca ML, Zarub M, Manconi M, Lai F, Valenti D, Sinico C, et al. Glycerosomes: A new tool for effective dermal and transdermal drug delivery. Int J Pharm 2013,455:66-74.

24. Ashtiani HRM, Bishe P, Lashgari NA, Nilforoushzadeh MA, Zare S. Liposomes in cosmetics. J Skin Stem Cell 2016;3:1-6.

25. Zhang K, Zhang Y, Li Z, Li N, Feng N. Essential oil-mediated glycerosomes increase transdermal paeoniflorin delivery: optimization, characterization, and evaluation in vitro and in vivo. Int J Nanomed 2017;12:3521-32.

26. Domenico L, Calandra P, Barreca D, Magazu S, Kiselev MA. Soft Interaction in Liposome Nanocarriers for Therapeutic Drug Delivery. Nanomaterials (Basel) 2016;6:125:1-26.

27. Manca ML, Peris JE, Melis V. Nanoincorporation of curcumin in polymer-glycerosomes and evaluation of their in vitroin vivo suitability as pulmonary delivery systems. RSC Adv 2015; 127:1-28.

28. Manca ML, Cencettib C, Matricardib P, Castangia I, Zaru M, Diez SC, et al. Glycerosomes: Use of hydrogenated soy phosphatidylcholine mixture and its effect on vesicle features and diclofenac skin penetration. Int J Pharm 2016;511:198204.

29. Nounou MI, El-Khordagui LK, Khalafallah NA, Khalil SA. Liposomal formulation for dermal and transdermal drug delivery: past, present and future. Recent Pat Drug Deliv Formul 2008;2:9-18.

30. Rani D, Singh C, Kumar A, Sharma VK. Formulation development and in vitro evaluation of minoxidil bearing glycerosomes. Am J Biomed Res 2016;4:27-37.

31. Quispe CAG, Coronado CJR, Carvalho JA. Glycerol: Production, consumption, prices, characterization and new trends in combustion. Rene Sustainable Energy Rev 2013;27:475-93.

32. Li Jing, Xuling W, Ting Z, Chunling W, Zhenjun H, Xiang L, et al. A review on phospholipids and their main applications in drug delivery systems. Asian J Pharm Sci 2015;10:81-98.

33. Kaddah S, Khreich N, Kaddah F, Charcosset C, Greige-Gerges H. Cholesterol modulates the liposome membrane fluidity and permeability for a hydrophilic molecule. Food Chem Toxicol 2018;113:40-48.

34. Bozzuto G, Molinari A, Liposomes as nanomedical devices. Int J Nanomed 2015; 10:975-99.

35. Needham D, Nunn RS. Elastic deformation and failure of lipid bilayer membranes containing cholesterol. Biophys J 1990;58:997-1009.

36. Gregoriadis G, Davis C. Stability of liposomes in vivo and in vitro is promoted by their cholesterol content in the presence of blood cells. Biochim Biophys Res Commun 1979;89:1287-93.

37. Kirby C, Gregoriadis G. Effect of the cholesterol content of small unilamellar liposomes on their stability in vivo and in vitro. Biochem J 1980;186:591-8.

38. Papahadjopoulos D, Jacobson K, Nir S, Isac T. Phase transitions in phospholipid vesicles. Fluorescence polarization and permeability measurements concerning the effect of temperature and cholesterol. Biochim Biophys 1973;311:33048.

39. Demel RA, De Kruyff B. The function of sterols in membranes. Biochim Biophys Acta 1976;457:109-32.

40. Virden JW, Berg JC. NaCl-induced aggregation of dipalmitoylphosphatylglycerol small unilamellar vesicles with varying amounts of incorporated cholesterol. Langmuir 1992;8:1532-15327. 
41. Liu DZ, Chen WY, Tasi LM, Yang SP. Microcalorimetric and shear studies on the effects of cholesterol on the physical stability of lipid vesicles. Colloids Surf 2000;172:57-67.

42. V.P. Torchilin, Recent advances with liposomes as pharmaceutical carriers. Nat Rev Drug Discov 2005;4:145160.

43. Eloya JO, Souzaa CDM, Petrilli R, Barcellos JPA, Lee RJ, Marchetti JM. Liposomes as carriers of hydrophilic small molecule drugs: strategies to enhance encapsulation and delivery. Colloids Surf B Biointerfaces 2014;1:345-63.

44. Akbarzadeh A, Sadabady RR, Soodabeh D, Joo SW, Zarghami N, Hanifehpour Y, et al, Liposome: classification, preparation, and applications. Nanoscale Res Lett 2013,8:1-9.

45. Laura MI, Franco D, Cattel L. Stealth liposomes: review of the basic science, rationale, and clinical applications, existing and potential. Int J Nanomed 2006;1:297-315.

46. Israelachvili JN. Aggregation of amphiphillic molecules into micelles, bilayers, vesicles and biological membranes. Intermolecular and Surface Forces. San Diego,CA, USA: Academic Press; 1991.

47. Sakai H, Gotoh T, Imura T, Sakai K, Otake K, Abe M. Preparation and properties of liposomes composed of various phospholipids with different hydrophobic chains usng a supercritical reverse phase evaporation method. J Oleo Sci 2008;57(11):613-21.

48. Lombardo D, Calandra P, Barreca D, Magazu S, A. Kiselev M. Soft interaction in liposome nanocarriers for therapeutic drug delivery. Nanomaterials 2016;6:1-26.

49. Salem HF, Kharshoum RM, Sayed OM, Hakim LFA Formulation design and optimization of novel soft glycerosomes for enhanced topical delivery of celecoxib and cupferron by box-behnken statistical design. Drug Development Ind Pharm 2018;44:1871-84.

50. M SAB, Yadav V, Mamatha Y, Prasanth VV. Liposomes: an overview. J Pharm Sci Innov 2012;1:13-21.

51. Patil YP, Jadhav S. Novel methods for liposome preparation. Chem Phys Lipids 2014;177:8-18.

52. Bangham AD, Standish MM, Weissmann G. The action of steroids and streptolysin S on the permeability of phospholipid structures to cations. J Mol Biol 1965;13:253-59.

53. Kim S, Jacobs RE, White SH. Preparation of multilamellar vesicles of defined size-distribution by solvent-spherule evaporation. Biochim Biophys Acta 1985;812:793-801.

54. Karami N, Moghimipour E, Salimi A. Liposomes as a novel drug delivery system: fundamental and pharmaceutical application. Asian J Pharm 2018;12:31-41.

55. Szoka F, Papahadjopoulos D. Procedure for preparation of liposomes with large internal aqueous space and high capture by reverse-phase evaporation. Proc Natl Acad Sci U S A 1978;75:4194-98.

56. Alpes H, Allmann K, Plattner H, Reichert J, Rick R, Shulz S. Formation of large unilamellar vesicles using alkyl maltoside detergents. Biochim Biophys Acta Biomembr 1986;862:294302.

57. Wang Q, Tan G, Lawson LB, John VT, Papadopoulos KD. Liposomes in double-emulsion globules. Langmuir 2010;6:3225-31.

58. Maheshkumar SS, Reddy KN, Goud PP, Kiranmayi N,
Arvind G. Formulation and characterization of doxorubicin hydrochloride liposomes by double emulsion method. Int Res J Pharma 2013;4:197-201.

59. Makhmalzadeh BS, Azh Z, Azarpanah A. Preparation and evaluation of mafenide acetate liposomal formulation as eschar delivery system. Int J Drug Development Res 2011;3:129-40.

60. Tiwari S, Verma P, Microencapsulation technique by solvent evaporation method (Study of effect of process variables). Int J Pharm Life Sci 2011;2:998-1005.

61. Deamer D, Bangham AD. Large volume liposomes by an ether vaporization method. Biochim Biophys Acta 1976;443:62934.

62. Laouini A. Preparation, characterization and applications of liposomes: state of the art. J Colloid Sci Biotech 2012;1:14768.

63. Batzri S, Korn ED. Single bilayer liposomes prepared without sonication. Biochim Biophys Acta 1973;298:1015-9.

64. Pons M, Foradada M, Estelrich J. Liposomes obtained by the ethanol injection method. Int J Pharm 1993;95:51-6.

65. Papahadjopoulos D, Vali WJ, Jacobson K, Poste G. Cochleate Lipid Cylinders: Formation by fusion of unilamellar lipid vesicles. Biochim Biophys Acta 1975;394:483-91.

66. Miller DC, Dahl GP. Early events in calcium-induced liposome fusion. Biochim Biophys Acta 1982;689:165-9.

67. Mayhew E, Lazo R, Vail WJ, King J, GreenAM. Characterization of liposomes prepared using a microemulsifier. Biochim Biophys Acta 1984;775:169-74.

68. Oku N, Macdonald RC. Formation of giant liposomes from lipids in chaotropic ion solutions. Biochim Biophys Acta 1983;734:54-61.

69. Pick U. Liposomes with a large trapping capacity prepared by freezing and thawing of sonicated phospholipid mixtures. Arch Biochem Biophys 1981;212:186-94.

70. Abdellatif MM, Khalil IA, Khalil MAF. Sertaconazole nitrate loaded nanovesicular systems for targeting skin fungal infection: in-vitro, ex-vivo and in vivo evaluation. Int J Pharm 2017;527:1-11.

71. Vitonyte J, Manca ML, Caddeo C. Bifunctional viscous nanovesicles co-loaded with resveratrol and gallic acid for skin protection against microbial and oxidative injuries. Eur J Pharm Biopharm 2017;114:278-287.

72. Manca ML, Manconi M, Zaru M, Valenti D, Peris JE, Matricardi $\mathrm{P}$, et al. Glycerosomes: investigation of role of 1,2-dimyristoyl-sn-glycero-3-phosphatidycholine (DMPC) on the assembling and skin delivery performances. Int J Pharm 2017;532:401-7.

73. Melis V, Manca ML, Bullita E. Inhalable polymer-glycerosomes as safe and effective carriers for rifampicin delivery to the lungs. Colloids Surf B Biointerfaces 2016;143:301-8.

74. Manconi M, Manca ML, Caddeo C, Sarais G, Palmieri A, Hallewin GD et al. Citrus lemon extract loaded in vesicular systems for the protection of oral cavity. Medicines (Basel). 2018;5:1-9.

75. Barichello JM, Yamakawa N, Kisyuku M, Handa H. Combined effect of liposomalization and addition of glycerol on the transdermal delivery of isosorbide 5-nitrate in rat skin. Int $\mathrm{J}$ Pharm 2008;357:199-205. 